\title{
Predicting Hyperkalemia by the Use of a 12-Lead Temporal-Spatial Electrocardiograph: Clinical Evaluations and Model Simulations
}

\author{
WC Tzeng, YZ Chan, JC Hsieh
}

Chung Hua University, Hsinchu, Taiwan

\begin{abstract}
Predicting hyperkalemia by the use of 12-lead electrocardiograph (ECG) is crucial in clinical emergency practice. However, the diagnostic sensitivity of hyperkalemia (serum potassium level > 5.0) is from $38 \%$ to $54 \%$ based on the interpretation of 12-lead ECG reports. In this study, a serial two-staged classifier implanted with K-means algorithm was created to screen hyperkalemia based on feature parameters extracted from 12-lead ECG. The SCP-ECG reports of 56 normal individuals and 41 hyperkalemia patients (serum potassium level > 5.3) were collected in the Emergency Department of Wei-Gong Memorial Hospital-Taiwan from January, 2003 through April, 2005. Each of the 12lead ECG reports was processed to generate two temporal-spatial graphs representing the signals on the limb leads and on chest leads, respectively. The two integrated T-wave volumes shown on the two graphs were selected as the feature parameters of the first staged classifier in screening hyperkalemia. The second staged classifier, which was composed of feature parameters such as PR interval, QRS duration, and QT interval, was used to correct the misclassification from the first stage. A computer program ECGSIM was also adopted to simulate the ECG waveforms from mild to severe hyperkalemia. Based on the results, the classifier showed the sensitivity of $85 \%(95 \% \mathrm{CI}=77 \%$ to $97 \%)$ and the specificity of $79 \%$ (95\% CI=55\% to 97\%) in classifying the samples. In conclusion, the two-staged classifier developed in this study shows the ability to extensively and efficiently diagnose patients with hyperkalemia from very mild to severe degrees. In addition, the estimated values of $T$-wave volumes from clinical data were in accordance with the observation of computer simulations, where $T$ volumes were increased with the increased serum potassium level.
\end{abstract}

\section{Introduction}

The rapidity and accuracy of predicting hyperkalemia was crucial in clinical practice. Hyperkalemia can be degenerated and endanger one's life if it is not diagnosed in time. Traditionally, the procedure for diagnosis of hyperkalemia is conducted through the laboratory blood test, which will take 20 minutes or more. Recently, the diagnosis of hyperkalemia can be conducted by the use of ECG because of its convenience and the possible correlations between potassium levels in serum and ECG features. The verification of the correlations between hyperkalemia and ECG may increase the diagnostic and treatment efficiency. However, the sensitivity of hyperkalemia prediction by ECG is very low (about $38 \%$ to $58 \%$ ) even by experienced cardiologists $[1,2]$, because the ECG features in response to hyperkalemia are still unclear. Based on recent clinical reports [3, 4], hyperkalemia is related to the changes of some ECG features. The peaked $\mathrm{T}$ waves could be observed in some chest leads or limb lead II in early hyperkalemia, and flatten $\mathrm{P}$ waves in some limb and chest leads. Other variables such as the increased PR interval, the QRS interval, and the RR interval could be seen in mild to severe hyperkalemia [5]. Nevertheless, the choice of the exact features in response to hyperkalemia is difficult to make because of the variability of the features. In our previous study [6], we developed a two-stage artificial neural network to predict patients' hyperkalemia (5.4 $7.4 \mathrm{mmole} / \mathrm{L})$. The results showed that the sensitivity of prediction reached $60 \%$ and the specificity was approximately $60 \%$. In this study, a serial two-staged classifier based on k-means method was developed to screen hyperkalemia using feature parameters extracted from 12-lead ECG. Furthermore, to search for better correlations between ECG features and hyperkalemia, a computer program ECGSIM was used to simulate the ECG waveforms under mild to severe hyperkalemic conditions.

\section{Methods}

\subsection{Extracting ECG feature parameters}

The SCP-ECG records of 56 normal individuals and 41 hyperkalemia patients (serum potassium level $>5.3$ ) were 
collected in the Emergency Department of Wei-Gong Memorial Hospital in Taiwan from January, 2003 through April, 2005. The collected SCP-ECG records were decoded to obtain the waveform data for each lead. The feature parameters of ECG were then extracted using a wavelet method. Temporal-spatial electrocardiographs representing the signals on the limb leads and on chest leads were constructed by interpolation using time, electric potential, and spatial location of limb leads or chest leads as axes as described by Chiang[8]. The Twave volumes of limb leads and chest leads, respectively.

\subsection{ECG feature selection}

Clinical cases indicated that peaked $\mathrm{T}$ waves in chest leads were usually observed in the early stage of mild hyperkalemia [3]. In addition, the widen QRS durations were usually reported in mild and severe hyperkalemic conditions [3]. According to electrocardiology, the increased potassium level in serum could result in slow action potential conduction. In ECG, the slowed conduction can be responsible for prolonged PR interval and QT interval [5]. The two integrated Twave volumes of each ECG were selected as the feature parameters of the first-stage classifier in screening hyperkalemia. The second-stage classifier, which was composed of feature parameters such as PR interval, QRS duration, and QT interval, was used to correct the misclassification from the first stage. The four features used to classify the hyperkalemic conditions were summarized in table 1 except the amplitude of $\mathrm{P}$ waves because of their small amplitudes in all leads.

Table 1. ECG features for various hyperkalemic conditions.

\begin{tabular}{|l|l|}
\hline Hyperkalemic condition & Possible features \\
\hline Mild & Peaked T waves \\
\hline \multirow{3}{*}{ Moderate } & $\begin{array}{l}\text { Prolonged PR interval, } \\
\text { Increased QTc, } \\
\text { Flatten P wave }\end{array}$ \\
\hline Severe & Widen QRS complex \\
\hline
\end{tabular}

\subsection{K-means based two-stage classifier}

97 cases, including 56 normal and 41 hyperkalemic individuals were collected. For each case, the T-wave volumes of limb leads and chest leads were used in the first-stage analysis; PR interval, QRS duration, and QT interval were used in the second-stage analysis. The collected ECG records were partitioned into $4 \quad(\mathrm{k}=4)$ clusters randomly based on the statistical observation of mean feature parameters listed in table 2. Each cluster was represented by its mean vector and each ECG record was classified into the cluster with the closed mean vector by iteration until there is no further change of error function. The calculation of mean vector is represented by equation (1), and error function is shown by equation (2).

$U_{i}=\frac{1}{n_{i}} \sum_{n=1}^{n} x_{n_{i}}$

$U_{i}$ represents the mean vector in $i$ th cluster, $n_{i}$ represents the number of the data in $i$ th cluster, and $x$ represents the data points in $i$ th cluster.

Error $=\sum_{i=1}^{k} \sum_{n \in S_{i}}\left\|x_{n_{i}}-u_{i}\right\|$

$k$ represents total partitioned clusters, $s_{i}$ represents the $i$ th cluster.

Based on the outputs of the K-means classifier [7], the input individual data will be categorized into one of four classifications. Only one out of the four classifications was used to represent the normal condition, the other three clusters were represented by hyperkalemia.

The two integrated T-wave volumes of limb leads and chest leads were selected as the feature parameters of the first-stage classifier in screening hyperkalemia. The second staged classifier, which was composed of feature parameters PR interval, QRS duration, and QT interval, was used to correct the misclassification from the first stage.

\subsection{Simulated ECG}

In order to identify the feature parameters of hyperkalemia ECG, the effects of potassium concentration in blood on the action potential of ventricle cells were studied by simulated ECG. First, the program "Human Ventricular Myocyte C++" was used to obtain conducting parameters of action potential (action potential duration and resting membrane potential) for heart cells under different potassium concentrations in blood. Potassium concentrations of 5.5, 6.5 and 7.5 $\mathrm{mmol} / \mathrm{L}$ were used to represent hyperkalemia and 4.0 $\mathrm{mmol} / \mathrm{L}$ was used to represent normal condition. The AP dispersions, including depolarization times, repolarization times and resting membrane potential, of 257 regions on the human surface of heart were calculated using the computer program ECGSIM from the action potential duration and resting membrane potential obtained above. The calculated AP dispersions were then used to perform simulation of electric potential of chest-lead and limblead on the body surface. The simulated 12-lead ECG and time-spatial ECG were constructed based on the results of the simulation. T-wave volumes of chest-lead and limb- 
lead were calculated from the simulated time-spatial ECG.

\section{Results}

\subsection{Statistical analysis}

The statistical mean values of selected feature parameters in normal cases and hyperkalemic cases were summarized in table 2. Both the limb-lead T-volume and chest-lead T-volume in hyperkalemia showed higher mean values and higher mean S.D. than normal cases. The mean values of PR interval, in both cases, were very close about $160 \mathrm{msec}$. However, the hyperkalemic case might display a larger dispersion in distribution because of its larger standard deviation (SD). The mean values of QTc were 107 with SD 38 vs. 91 with SD 32 in hyperkalemia and normal cases respectively. Obviously, the QTc could be a better index in the classification. Similarly, the QRS duration in hyperkalemia had a higher mean value, $107 \mathrm{msec}$ than $91 \mathrm{msec}$ of normal case, but also the higher value of SD in hyperkalemia indicating the clustering dispersion in hyperkalemia.

Table 2. The mean values and standard deviations (in parenthesis) of ECG parameters used in k-means analysis

\begin{tabular}{|l|c|c|}
\hline $\begin{array}{l}\text { Feature } \\
\text { parameter }\end{array}$ & Normal $^{\mathrm{a}}$ & Hyperkalemia $^{\mathrm{a}}$ \\
\hline $\begin{array}{c}\text { T-volume } \\
\text { (Limb leads) }\end{array}$ & $0.072(0.037)$ & $1.828(1.635)$ \\
\hline $\begin{array}{c}\text { T-Volume } \\
\text { (Chest leads) }\end{array}$ & $0.091(0.052)$ & $2.047(1.240)$ \\
\hline PR interval & $161.73(28.20)$ & $161.75(36.75)$ \\
\hline QTc & $404.19(32.54)$ & $425.11(38.48)$ \\
\hline QRS Duration & $91.00(13.87)$ & $107.20(26.26)$ \\
\hline
\end{tabular}

56 of normal cases and 41 of hyperkalemia cases

\subsection{K-means clustering analysis}

The outcome of k-means based two-stage classifier was summarized in table 3. Based on the results of automatic classification, the classifier showed the sensitivity of $85 \%(95 \% \mathrm{CI}=77 \%$ to $97 \%)$ and a specificity of $79 \%$ (95\% CI=55\% to $97 \%$ ) in classifying the samples.
Table 3. The sensitivity and specificity of the k-means classifier in screening hyperkalemia.

\begin{tabular}{|c|c|c|}
\hline & Percentage(\%) & $\begin{array}{c}95 \% \text { confidence } \\
\text { interval }(\%)\end{array}$ \\
\hline Sensitivity & 85 & $77-97$ \\
\hline Specificity & 79 & $55-97$ \\
\hline
\end{tabular}

\subsection{Simulated ECG}

The results of simulation showed that $\mathrm{T}$-wave volumes of chest-lead and limb-lead were 1.176 and 1.513, respectively, when potassium concentrations was 5.5 $\mathrm{mmol} / \mathrm{L} ; 1.585$ and 1.322 when potassium concentrations was $6.5 \mathrm{mmol} / \mathrm{L} ; 1.987$ and 1.873 when potassium concentrations was $7.5 \mathrm{mmol} / \mathrm{L} ; 0.673$ and $1.513 \mathrm{mmol} / \mathrm{L}$ when potassium concentrations was set at the normal concentration. Compared with clinical data, the simulation showed the same trend for the relationship of T-wave volume and potassium concentration of hyperkalemia patients. T-wave volume increased as potassium concentration increased (table 4).

Table 4. Comparison of T-volumes obtained from simulated ECG and clinical ECG.

\begin{tabular}{|c|c|c|c|c|}
\hline \multirow{2}{*}{$\begin{array}{c}{[\mathrm{K}+]} \\
\mathrm{mmol} / \mathrm{L}\end{array}$} & \multicolumn{2}{|c|}{ Simulated ECG } & \multicolumn{2}{c|}{ Clinical ECG } \\
\cline { 2 - 5 } & Limb & Chest & Limb & Chest \\
\hline Normal & 1.513 & 0.673 & 0.072 & 0.091 \\
\hline $5.5^{\mathrm{a}}$ & 1.531 & 1.176 & 1.271 & 1.543 \\
\hline $6.5^{\mathrm{b}}$ & 1.332 & 1.585 & 1.992 & 2.179 \\
\hline $7.5^{\mathrm{c}}$ & 1.873 & 1.987 & 2.523 & 2.711 \\
\hline
\end{tabular}

$[\mathrm{K}+]=5.4-5.9 \mathrm{mmol} / \mathrm{L}$ for clinical $\mathrm{ECG}$

$[\mathrm{K}+]=6.0-6.9 \mathrm{mmol} / \mathrm{L}$ for clinical $\mathrm{ECG}$

$[\mathrm{K}+]=7.0 \mathrm{mmol} / \mathrm{L}$ and up for clinical $\mathrm{ECG}$

\section{Discussion and conclusions}

The dimension of classification was decreased from the previous 17 to 5 in this study [6]. The benefits of the reduced dimension model were not only to reduce the complexity of classification but also to speed up the iteration in the algorithm of k-means. The PR interval, QTc, and QRS duration were used in the previous model except the two T-volumes of limb leads and chest leads. The two T-volumes were derived from the time-spatial electrocardiograph constructed from data of traditional ECG. The overall performance of the proposed model was greatly increased in sensitivity from $60 \%$ to $85 \%$ and the specificity was increased from $60 \%$ to $79 \%$. From statistical analysis, the features in hyperkalemia showed larger SD than normal cases. Accordingly, we concluded 
that this is the feature showing clearly the differences between normal cases and hyperkalemia, which were not clear by the traditional methods. Furthermore, the results of simulated ECG indicated that the $\mathrm{T}$ wave volume may be used as an important characteristic for diagnosing hyperkalemia. Based on clinical report, the sensitivity of hyperkalemia prediction from E.C.G. was about $38 \%$ by experienced cardiologists while the potassium level was below $6.5 \mathrm{mmol} / \mathrm{L}$ [1]. The sensitivity was increased to $58 \%$ when potassium level in serum was greater than 6.5 $\mathrm{mmol} / \mathrm{L}$. The specificity in the same test was about $85 \%$ when potassium level was greater than $5 \mathrm{mmol} / \mathrm{L}$. Based on the results of the k-means based classifier, the classifier had a sensitivity of $85 \%(95 \% \mathrm{CI}=77 \%$ to $97 \%)$ and a specificity of $79 \%(95 \% \mathrm{CI}=55 \%$ to $97 \%)$. It should be noted that the proposed method can greatly promote the sensitivity of hyperkalemia prediction by ECG. In this study, the k-means based classifier can be used as a potential screening test, because of its increased sensitivity.

\section{Acknowledgements}

This project is granted by NSC (National Science Council, NSC 94-2213-E-216-011 and NSC 94-2213-E216-030), Taiwan. Our gratitude is extended to ShihMing Shieh, M.D., the head of Wei-Gong Memorial Hospital, who generously assisted our data collection. Finally, we thank for the funding and support provided by Chung Hua University, Taiwan.

\section{References}

[1] Wrenn KD etc. The ability of physicians to predict hyperkalemicnfrom the ECG. Ann Emerg Med. 1991; 20:1229-1232.

[2] Dreifus LS etc. A clinical correlative study of the electrocardiogram in electrolyte imbalance. Circulation. 1956; 14: 815-825.

[3] Slovis C. etc. ABC of clinical electrocardiography. BMJ 2002;324:1320-1323.

[4] Macfarlane PW etc. Comprehensive Electrocardiology. New York, Pergamon Press, 1989.

[5] Poters RS etc. Prediction of hyperkalemia in dogs from electrocardiographic parameters using an artifificial neural network. Academic Emergency Medicine. 2001; 8(6):599603.

[6] Wu MF, Tzeng WC, Hsieh JC etc. Predicting hyperkalemia based on a two stage artificial neural network. Computers in Cardiology, 2003; 30:433-436.

[7] Nabney IT. Netlab Algorithms for pattern recognition. pp 101-104. Springer, 2001.

[8] Chiang HK et al: A new 3-D display method for 12-lead ECG. IEEE Trans. Biomed Eng. 2001; 48:1195-1201.
Address for correspondence

Jui-Chien Hsieh, Ph.D.

Department of Bioinformatics

Chung Hua University

No.707, Sec.2, WuFu Rd.

Hsinchu, Taiwan 30067, R.O.C.

E-mail address : jchsieh@chu.edu.tw 